\title{
Imputation of microsatellite alleles from dense SNP genotypes for parental verification
}

\author{
Matthew McClure ${ }^{1}$, Tad Sonstegard ${ }^{1}$, George Wiggans ${ }^{2}$ and Curtis P Van Tassell ${ }^{1}$ * \\ Bovine Functional Genomics Lab, United States Department of Agriculture, Agriculture Research Service, Beltsville, MD, USA \\ ${ }^{2}$ Animal Improvement Program Lab, United States Department of Agriculture, Agriculture Research Service, Beltsville, MD, USA
}

\author{
Edited by: \\ Jean Michel Claude Elsen, Institut \\ National de la Recherche \\ Agronomique, France \\ Reviewed by: \\ Juan Steibel, Michigan State \\ University, USA \\ Alexandre Rodrigues Caetano, \\ Embrapa Recursos Genéticos e \\ Bioteclogia, Brazil \\ *Correspondence: \\ Curtis P Van Tassell, Bovine Functional \\ Genomics Lab, United States \\ Department of Agriculture, \\ Agriculture Research Service, 10300 \\ Baltimore Avenue, Building 200, \\ Beltsville, MD 20705-2350, USA. \\ e-mail: curt.vantassell@ars.usda.gov
}

Microsatellite (MS) markers have recently been used for parental verification and are still the international standard despite higher cost, error rate, and turnaround time compared with Single Nucleotide Polymorphisms (SNP)-based assays. Despite domestic and international interest from producers and research communities, no viable means currently exist to verify parentage for an individual unless all familial connections were analyzed using the same DNA marker type (MS or SNP). A simple and cost-effective method was devised to impute MS alleles from SNP haplotypes within breeds. For some MS, imputation results may allow inference across breeds. A total of 347 dairy cattle representing four dairy breeds (Brown Swiss, Guernsey, Holstein, and Jersey) were used to generate reference haplotypes. This approach has been verified (>98\% accurate) for imputing the International Society of Animal Genetics recommended panel of $12 \mathrm{MS}$ for cattle parentage verification across a validation set of 1,307 dairy animals. Implementation of this method will allow producers and breed associations to transition to SNP-based parentage verification utilizing MS genotypes from historical data on parents where SNP genotypes are missing. This approach may be applicable to additional cattle breeds and other species that wish to migrate from MS- to SNP-based parental verification.

Keywords: microsatellite imputation, parentage verification, SNP haplotype, ISAG, across breed imputation

\section{INTRODUCTION}

Microsatellite markers (MS) have successfully been used for parentage verification in multiple livestock species over the past few decades. Their impact on the industry for ensuring accurate pedigree information has been immense, both for parental verification of registered animals and parental identification in multi-sire pastures (Davis and Denise, 1998; Gomez-Raya et al., 2008). Pedigree errors bias estimates of heritability, breeding values, estimates of genetic parameters, prediction of genetic gain, and depress the rate of genetic progress from selection of superior breeding animals (Israel and Weller, 2000; Senneke et al., 2004; Harlizius et al., 2011). While MS have a high polymorphic information content (PIC), allele scoring is difficult to fully automate with high accuracy because of preferential allele amplification, imperfect repeats, null alleles, and allelic dropouts (Kelly et al., 2011). Furthermore, manual scoring is prone to human error due to the complexity of patterns and data anomalies such as appearance of stutter bands (Baruch and Weller, 2008; Kelly et al., 2011). It has been estimated that MS error rate for allele scoring ranges from 1 to $5 \%$ (Baruch and Weller, 2008) to more than $30 \%$ per locus (Gagneux et al., 1997). While multiplexing can help reduce time and cost of MS panels, such as the International Society of Animal Genetics' (ISAG) parental verification panels ${ }^{1}$, it can be extremely challenging to properly optimize amplification conditions to minimize error rates (Luikart et al., 2008; McClure et al., 2010).

${ }^{1}$ http://bit.ly/ISAG_pv
In comparison to MS, SNP have a lower PIC due to their bi-allelic nature, but there is increasing interest from both the production agriculture and research communities to use SNP for parental verification. Advantages of SNP genotyping include minimal human interaction, lower error rates, ease of automation, and standardization between laboratories (Heaton et al., 2002; Anderson and Garza, 2006; Van Eenennaam et al., 2007; Baruch and Weller, 2008). Additionally, the genotyping cost for parental verification can be greatly reduced by using SNP, in place of MS markers. For example, Tokarska et al. (2009) estimated that using the Veracode system (Illumina Inc, San Diego, CA, USA) 50 bovine SNP can be genotyped for $1 / 2$ the cost of 16 MS. If a set of parentage SNP were integrated into larger genotyping panels the overall price of the parental verification SNP would further decrease. This approach was employed with a set of 121 parentage SNP that were selected from a large number of candidate SNP using call-rate and allele frequencies across a large number of breeds (Heaton et al., 2002). These markers have been incorporated into all commercially available genotyping assays, including Illumina's BovineSNP50 (Matukumalli et al., 2009) BovineHD (Illumina Inc, 2010), Bovine3K (Illumina Inc, 2011), and BovineLD (Boichard et al., 2012) and the Affymetrix BOS 1 (Affymetrix Inc, 2011).

Despite the advantages of SNP panels in cost, accuracy, and automation, transition from MS to SNP markers for parentage verification has been very slow. A major hindrance to this transition is the need for duplicate genotyping during this transition period as both parents and offspring must be genotyped with the same technology for parental verification. This requirement 
is a major limitation for historic animals where a DNA source does not exist due to culling, death, or change in ownership of animals. Furthermore, the additional cost of MS genotyping is difficult to justify when increasing numbers of commercial dairy cows are SNP genotyped and nearly every dairy sire is SNP genotyped. To address this issue of transition genotyping, a simple and cost-effective method is proposed to impute MS alleles from SNP haplotypes. This strategy may be implemented in any species that has dense SNP genotypes and MS alleles on a large enough subset of the population to determine phase relationships between MS alleles and SNP haplotypes.

\section{MATERIALS AND METHODS ANIMALS}

Illumina Bovine High Density SNP (BovineHD; Illumina Inc, 2010) genotypes for dairy cattle $(N=1,301)$ were obtained from the industry database maintained by the Animal Improvement Programs Laboratory (AIPL) of the United States Department of Agriculture's Agricultural Research Service (USDA-ARS) ${ }^{2}$. Animals from the USA represented four breeds Brown Swiss (BS, $n=71$ ), Guernsey (GU, $n=60)$, Holstein (HO, $n=1,110$ ), and Jersey (JE, $n=60$; Table 1 ).

\section{GENOTYPES}

The International Society of Animal Genetics has become the de facto authority organization for parentage testing. The current list of MS markers was established nearly 20 years ago (Baumung et al., 2004). Genotypes for the ISAG-sanctioned 12 MS bovine panel (BM1818, BM1824, BM2113, ETH3, ETH10, ETH225, INRA023, SPS115, TGLA53, TGLA122, TGLA126, TGLA227) $)^{3}$ for offspring and their parents were obtained from the respective breed associations [US Brown Swiss Association (USBSA), USA Holstein Association (HAUSA), US Jersey Association (USJA), American Guernsey Association]. Locations of the MS on the UMD3.1 bovine genome assembly (Zimin et al., 2009) were determined by using BLAST (Altschul et al., 1990) to align reported primer sequences (Table 2 ).

\footnotetext{
${ }^{2} \mathrm{http}: / /$ www.marc.usda.gov/genome/cattle/cattle.html
}

${ }^{3}$ http://www.isag.us/Docs/CattleMMPTest_CT.pdf
BovineHD SNP genotypes for markers within $500 \mathrm{~kb}$ of each MS (average 301 SNP) were obtained from USDA-ARS AIPL. These genotypes represent animals from the HapMap population; animals genotyped in research projects at the USDA-ARS Bovine Functional Genomics Laboratory (see text footnote 2); and animals with data exchanged as part of Cooperative Dairy DNA Repository Steering Committee's collaborations with the Canadian Dairy Network, DairyCo of the United Kingdom, and ANAFI of Italy. SNP data was in AB format from GenomeStudio (Illumina).

All genotype data was loaded into SVS7 [SNP and Variation Suite v7.5; (Golden Helix, Bozeman, MT, USA]. Minor allele frequencies (MAF) at all loci were required to exceed 5\% across breeds.

\section{HAPLOTYPE IDENTIFICATION}

The BEAGLE program (Browning and Browning, 2007) was used to phase the genotypes, as this software was one of the few that could handle both bi- and multi-allelic data. SNP loci used for phasing and the DNA base associated with "A" and "B" alleles are listed in Table S1 in Supplementary Material. Genotypic data for 347 animals (33 BS, 16 GU, 250 HO, 48 JE, Table 1) that had both MS and flanking SNP data (the "combined" data set) was exported from SVS7, and each breed was independently phased by BEAGLE using 1,000 iterations.

Haplotypes were initially determined using 10 SNP and the MS genotype centered on a MS. Haplotypes of increasing sizes were analyzed by counting the number of times each MS allele was phased with each SNP haplotype (MS-haplotype). The extension of a haplotype was ended when a haplotype size was identified that sufficiently fit the following conditions:

1. Minimize the number of MS alleles associated with a haplotype within a breed (ideally, each haplotype associated with a single MS allele),

2. Minimize the number of singular MS-haplotype association counts,

3. Minimize the number of haplotypes with multiple MS alleles associated with a single haplotype,

4. Minimize the total number of SNP needed to impute MS alleles.

Table 1 | Animal and genotype count.

\begin{tabular}{|c|c|c|c|c|c|c|c|}
\hline Breed $^{a}$ & HDSNPb & \multicolumn{6}{|c|}{ Microsatellite $^{c}$} \\
\hline GU & 60 & 0 & 5 & 5 & 6 & 2 & 0 \\
\hline $\mathrm{HO}$ & 1110 & 6 & 49 & 118 & 77 & 395 & 0 \\
\hline JE & 60 & 3 & 8 & 15 & 22 & 57 & 26 \\
\hline
\end{tabular}

${ }^{a}$ Breed abbreviations: BS, Brown Swiss; GU, Guernsey; HO, Holstein; JE, Jersey.

${ }^{b}$ Count of individuals with HDSNP genotypes.

${ }^{\circ}$ Count of individuals with genotypes for $9,10,11$, or 12 microsatellite loci.

${ }^{d}$ Count of sires with marker genotypes from microsatellites.

${ }^{e}$ Count of dams with marker genotypes from microsatellites. 


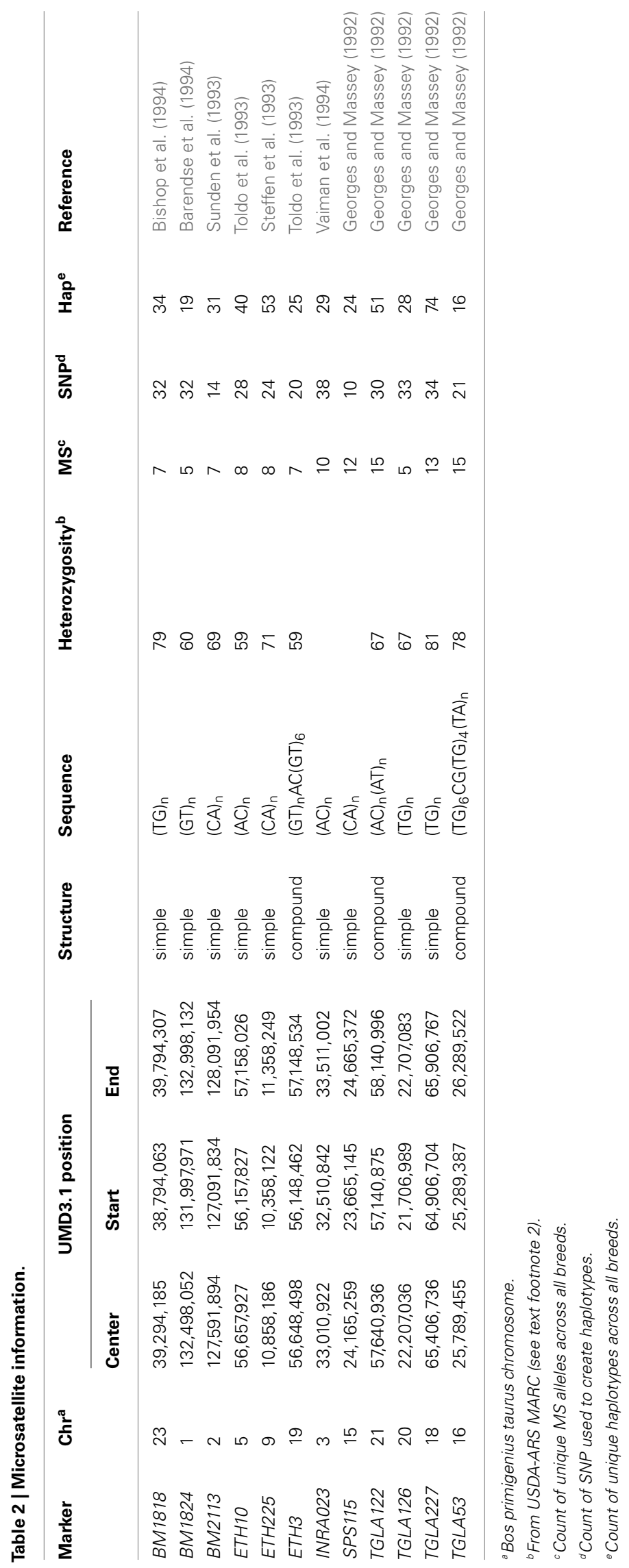


Table 3 illustrates the BM1824 allele counts per haplotype for the 32 SNP haplotype that satisfy the above criteria. The resulting counts were condensed into an MS imputation table (Table S2 in Supplementary Material) showing the fraction of MS alleles observed with each haplotype by breed.

\section{WITHIN BREED IMPUTATION}

Genotypes for the SNP from haplotypes identified above (Table S2 in Supplementary Material) were independently phased by breed (BS, GU, HO, and JE) for 1,301 animals in BEAGLE. The 347 animals used to generate the haplotypes were a subset of the 1,301. The resulting SNP haplotypes were then compared to those determined using the combined SNP and MS genotypes and listed in the breed specific imputation table (Table S2 in Supplementary Material). For novel SNP haplotypes not observed in the combined data, no MS allele was inferred. As a result of these non-predicted genotypes, an animal could have two, one, or no MS alleles imputed. Only MS-haplotype information within breeds was used to impute MS alleles.

\section{ACROSS BREED IMPUTATION}

When multiple MS alleles were associated with a haplotype within a breed the less frequent allele was rarely observed over 2 times. While multiple MS alleles could be associated with a haplotype because of higher mutation rates of MS compared with SNP $\left(4.5 \times 10^{-5}\right.$ vs. $1 \times 10^{-5}$; Ellegren, 1995 ; Falconer and Mackay, 1996), there is a much higher chance that this discrepancy resulted from MS genotyping errors. To better account for low-frequency improper assignments of MS alleles with SNP haplotypes, an alternative MS imputation was devised where a MS-haplotype association had to be observed a minimum four times for that association to be considered for MS imputation.

Analysis of the imputation table (Table S2 in Supplementary Material) also revealed that MS-haplotype combinations often held true across breeds. To assess if MS-haplotype combinations were present before modern breed formations, and have been preserved across breeds, MS-haplotype information from other breeds was used to impute alleles if the haplotype was not observed in the breed during the initial haplotype identification.

\section{VALIDATION}

Imputed and breed association reported MS genotypes were compared as follows: (1) parentage verification using only reported MS, (2) parentage verification using individual imputed MS and observed parental MS genotypes, and (3) concordance between the reported and imputed MS genotype. When multiple MS alleles were associated with a SNP haplotype, all associated MS alleles were considered as possible alleles in the analysis. Parentage verification was performed by locus and failed if two putative MS allele could not have been inherited from either parent.

\section{RESULTS}

For all MS loci tested, the 424 SNP-based haplotypes identified from 316 SNP (Tables S1 and S2 in Supplementary Material) effectively identified MS alleles for the analyzed breeds. When using the within breed imputation method 358 haplotypes were associated with a single MS allele, 50 of these unique associations were observed in at least two breeds. A total of 165 of these breed specific associations were seen only in Holstein, possibly because of the larger data set for that breed. While 358 haplotypes were associated with one MS allele, 11 were associated with a single MS allele within a breed and multiple alleles across breeds, with the remaining 55 associated with multiple MS alleles in at least one breed. Considering the numbers of genotypes in the combined data set, these counts were $5,945,260$, and 1,487 , respectively.

With the across breed imputation method 384 haplotypes were associated with a single MS allele, 65 of these being observed in at least two breeds. For the remaining haplotypes: 21 were associated with single MS allele within a breed and multiple alleles across breeds, and 19 were associated with multiple MS alleles in at least one breed. Considering the numbers of genotypes in the combined data set, these counts were 6,366, 498, and 828, respectively.

While using only within breed imputation resulted in a slightly higher concordance rate between an individual's reported and imputed MS allele (99.4 vs. 98.0\%) 334 more genotypes were imputed using the across breed imputation method across the 1,301 individuals (Table 4 and Table S3 in Supplementary Material). On a per locus basis, parentage verification rates were $99.8 \%$ for MS reported alleles, $99.4 \%$ for within breed imputed MS alleles, and $99.2 \%$ for across breed imputed alleles. Statistically, these rates are not different $(P>0.13$; Table 5).

\section{DISCUSSION}

Techniques must be developed to bridge traditional and new technologies in order to allow producers to benefit from cheaper parentage assays while maintaining compatibility with previous historic data; thus avoiding additional costs of re-genotyping the parental generation. The method developed here represents an effective and inexpensive way for the livestock genetics community to parentally verify an individual when separate genotyping platforms have been used across the generations. There are currently a large number of active North American dairy cattle that could use MS imputation for parentage verification. The HAUSA currently uses both SNP- and MS-based methods for parental verification, and from 3/2011-3/2012 845 bulls and 7,468 dams (out of a total of 8,643 and 236,406 respectively) were verified using MS only (Tom Lawlor-HAUSA, Personal communication 3/19/2012). In a similar manner the USJA has 1,918 dams in the breeding herd that only have MS genotypes (Erick Metzger-USJA, Personal communication 3/27/2012). Starting in 2012 the USJA has begun using only SNP for parentage verification. MS imputation would enable USJA, and others (Dave Kindall-USBSA, Personal communication $4 / 4 / 2012$ ) to eliminate the need to re-test these dams with SNP. For researchers this method will allow for the merging of historic and current data even though they are derived from different types of markers.

Scant literature exists that report MS genotyping error rates, but Bonin et al. (2004) estimated an error rate of $0.8 \%$ per allele in bears. These "errors often go undetected because they are generally unobtrusive" (Bonin et al., 2004). A random set of genotyping errors were not present in this study, because the genotypes used were those confirming parentage. If a majority of marker genotypes provide support for a parent-progeny relationship, then the 


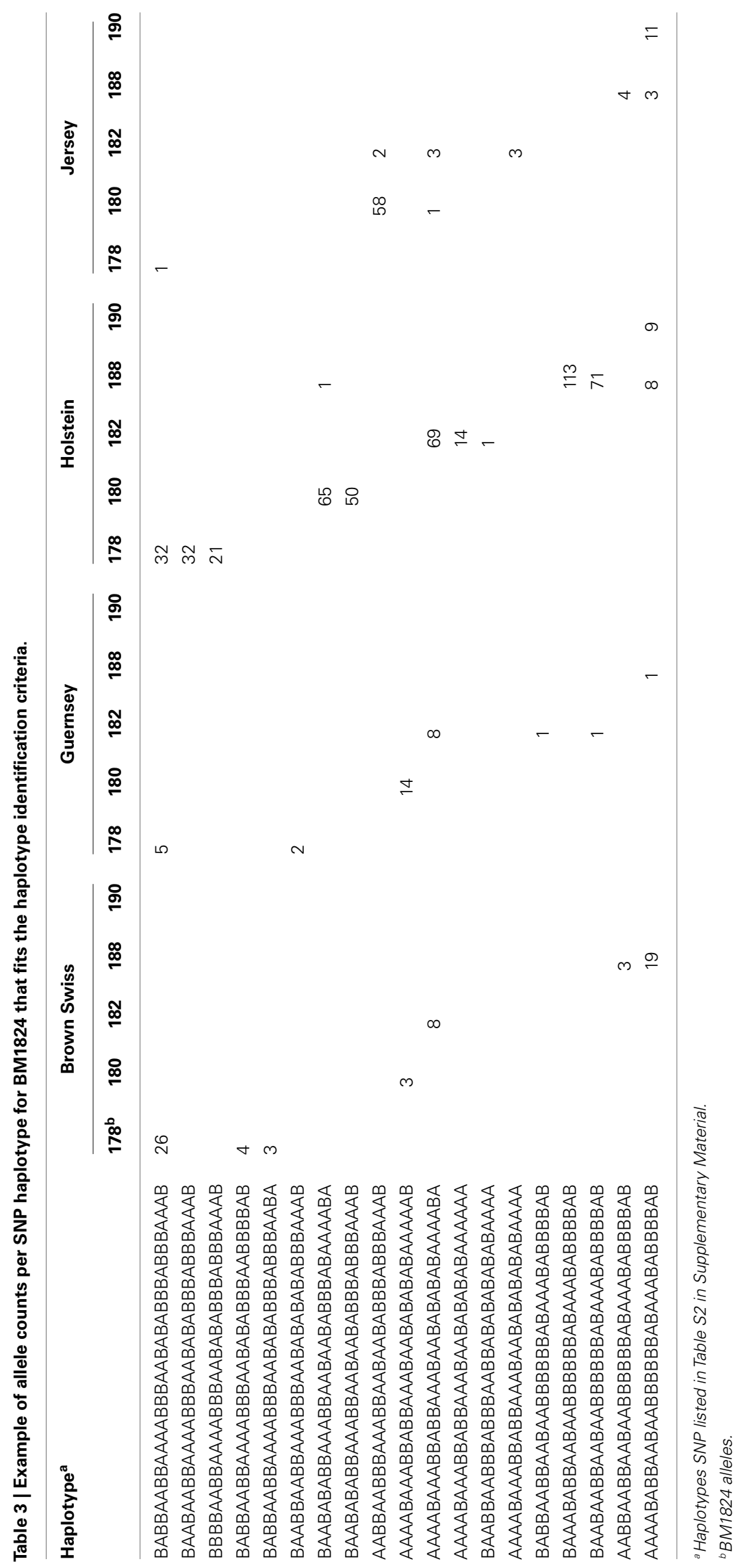


Table 4 | Percent of 1,301 individuals with haplotypes imputed to microsatellite (MS) alleles.

\begin{tabular}{|c|c|c|c|c|c|c|c|}
\hline \multirow[t]{2}{*}{ MS } & \multirow[t]{2}{*}{ Alleles $^{a}$} & \multicolumn{2}{|c|}{0 Allele } & \multicolumn{2}{|c|}{1 Allele } & \multicolumn{2}{|c|}{2 Allele } \\
\hline & & Withinc & Across $^{d}$ & Within & Across & Within & Across \\
\hline BM1818 & 7 & 0.020 & 0.012 & 0.980 & 0.988 & 0.834 & 0.858 \\
\hline$B M 1824$ & 5 & 0.001 & 0.001 & 0.999 & 0.999 & 0.983 & 0.988 \\
\hline$B M 2113$ & 7 & 0.004 & 0.001 & 0.996 & 0.999 & 0.960 & 0.985 \\
\hline ETH10 & 8 & 0.010 & 0.003 & 0.99 & 0.997 & 0.940 & 0.949 \\
\hline ETH225 & 8 & 0.037 & 0.018 & 0.963 & 0.982 & 0.902 & 0.932 \\
\hline ETH3 & 7 & 0.005 & 0.005 & 0.995 & 0.995 & 0.947 & 0.957 \\
\hline INRA023 & 10 & 0.012 & 0.002 & 0.988 & 0.998 & 0.947 & 0.961 \\
\hline SPS115 & 7 & 0.006 & - & 0.994 & 1.000 & 0.961 & 0.988 \\
\hline TGLA122 & 15 & 0.044 & 0.017 & 0.956 & 0.982 & 0.891 & 0.904 \\
\hline TGLA126 & 5 & 0.015 & 0.009 & 0.985 & 0.991 & 0.944 & 0.960 \\
\hline TGLA227 & 13 & 0.033 & 0.028 & 0.967 & 0.972 & 0.889 & 0.892 \\
\hline TGLA53 & 15 & 0.002 & - & 0.998 & 1.000 & 0.954 & 0.971 \\
\hline Average & 8.9 & 0.016 & 0.008 & 0.984 & 0.992 & 0.929 & 0.945 \\
\hline
\end{tabular}

${ }^{a}$ Number of unique MS alleles.

${ }^{\circ} 0=M S$ alleles were not imputed from either haplotype, $1=M S$ allele imputed from only 1 haplotype, $2=M S$ alleles imputed from both haplotypes.

${ }^{c} \mathrm{MS}$ imputation performed only using within breed haplotype data.

${ }^{d} \mathrm{MS}$ imputation performed using across and with breed haplotype data.

Table 5 | Microsatellite (MS) validations.

\begin{tabular}{|c|c|c|c|c|c|c|c|c|c|c|c|c|c|c|}
\hline \multirow[t]{4}{*}{ Microsatellite } & \multicolumn{3}{|c|}{ Concordance $^{a}$} & \multicolumn{11}{|c|}{ Parentage verification ${ }^{b}$} \\
\hline & \multirow[t]{3}{*}{ Withing } & \multirow[t]{3}{*}{ Across $^{h}$} & \multirow[t]{3}{*}{$N^{i}$} & \multicolumn{2}{|c|}{ Reported MSc } & \multicolumn{9}{|c|}{ Imputed MS } \\
\hline & & & & & $N$ & \multicolumn{3}{|c|}{ Overall } & \multicolumn{3}{|c|}{ Not reported ${ }^{\mathrm{e}}$} & \multicolumn{3}{|c|}{ Reported $^{f}$} \\
\hline & & & & & & Within & Across & $N$ & Within & Across & $N$ & Within & Across & $N$ \\
\hline BM1818 & 0.993 & 0.977 & 133 & 1.000 & 37 & 0.972 & 0.979 & 144 & 0.991 & 0.991 & 107 & 0.921 & 0.947 & 38 \\
\hline$B M 1824$ & 1.000 & 0.994 & 337 & 0.995 & 189 & 1.000 & 1.000 & 475 & 1.000 & 1.000 & 289 & 1.000 & 1.000 & 188 \\
\hline$B M 2113$ & 0.991 & 0.980 & 344 & 1.000 & 195 & 0.998 & 0.998 & 478 & 1.000 & 1.000 & 284 & 0.995 & 0.995 & 194 \\
\hline ETH10 & 1.000 & 0.988 & 343 & 1.000 & 194 & 0.996 & 0.987 & 479 & 0.997 & 0.986 & 285 & 0.995 & 0.990 & 195 \\
\hline ETH225 & 0.988 & 0.955 & 325 & 1.000 & 154 & 0.991 & 0.979 & 470 & 0.989 & 0.975 & 283 & 0.995 & 0.984 & 187 \\
\hline ETH3 & 0.997 & 0.991 & 340 & 1.000 & 149 & 1.000 & 1.000 & 479 & 1.000 & 1.000 & 288 & 1.000 & 1.000 & 192 \\
\hline INRA023 & 0.991 & 0.988 & 343 & 0.990 & 194 & 1.000 & 1.000 & 479 & 1.000 & 1.000 & 285 & 1.000 & 1.000 & 194 \\
\hline SPS115 & 0.979 & 0.954 & 340 & 1.000 & 195 & 0.996 & 0.994 & 479 & 0.997 & 1.000 & 284 & 0.995 & 0.985 & 196 \\
\hline TGLA122 & 0.997 & 0.959 & 319 & 1.000 & 195 & 0.996 & 0.979 & 471 & 0.993 & 0.986 & 282 & 1.000 & 0.958 & 189 \\
\hline TGLA126 & 0.997 & 0.988 & 345 & 1.000 & 194 & 1.000 & 1.000 & 478 & 1.000 & 1.000 & 284 & 1.000 & 1.000 & 194 \\
\hline TGLA227 & 1.000 & 0.979 & 337 & 1.000 & 189 & 0.993 & 0.989 & 475 & 0.990 & 0.990 & 286 & 1.000 & 0.989 & 190 \\
\hline TGLA53 & 1.000 & 0.970 & 297 & 0.987 & 153 & 0.991 & 0.993 & 431 & 1.000 & 1.000 & 279 & 0.974 & 0.980 & 152 \\
\hline Average & 0.994 & 0.977 & 317 & 0.998 & 170 & 0.994 & 0.992 & 445 & 0.996 & 0.994 & 270 & 0.989 & 0.986 & 176 \\
\hline
\end{tabular}

${ }^{a}$ Percent of imputed MS allele(s) match the reported alleles.

${ }^{b}$ Percent of individuals per locus that pass parentage verification.

cParentage verification using only breed reported MS alleles on the individual and its parents.

${ }^{d}$ Parentage verification using imputed MS alleles on the individual and breed reported parental MS alleles.

e Individuals did not have a breed reported MS allele.

${ }^{f}$ Individuals that had a breed reported MS allele.

${ }^{g} \mathrm{MS}$ imputation performed only using within breed haplotype data.

${ }^{h} \mathrm{MS}$ imputation performed using across and with breed haplotype data.

${ }^{i}$ Count of individual. 


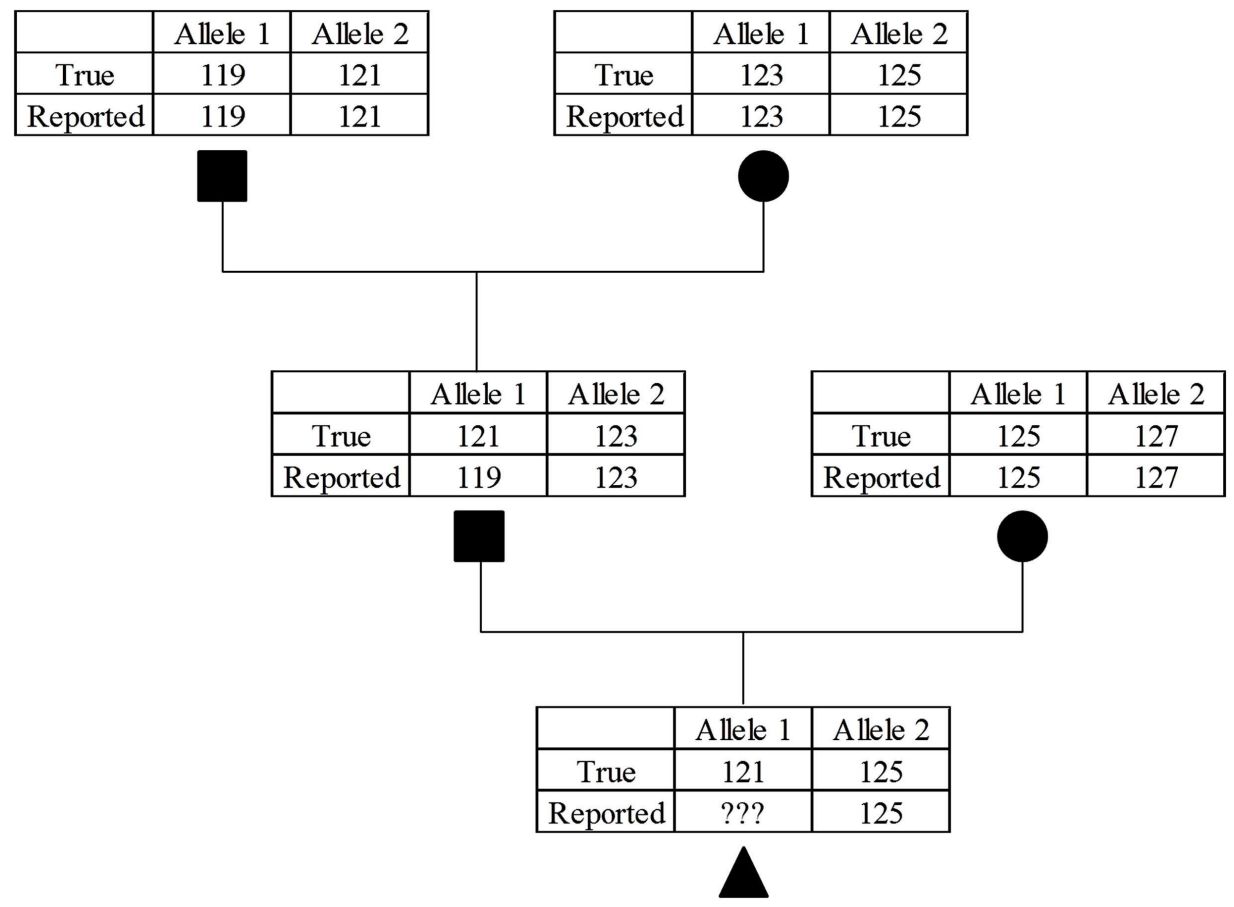

FIGURE 1 | Example of the compounding effect of MS allele miscalls. While the sire in the second generation was misgenotyped for the parental allele it was not caught because the error still follows Mendelian inheritance patterns. This will cause a problem in the third generation because if the parental 121 allele is called correctly it will fail the parentage verification, if incorrectly called again as a 119 allele this error will further propagate in future generations. genotypic data would likely be revisited and potentially revised. This problem can be envisioned by considering a case where a bull had an improperly scored allele for a MS genotype which did not violate Mendelian inheritance (Figure 1). Depending on the genotype of the bull's mate, this situation may result in forcing the genotype to be incorrect to avoid non-inheritance of MS alleles. This situation could be problematic in the vignette described, because the bull's genotypes would precede his offspring by a considerable time, and the sire's genotype would be considered correct (and quite possibly no DNA available for retesting). A practical consideration is that it is likely that the same chemistry conditions that led to the original allelic dropout or false alleles would occur again. Regardless of the cause, these genotyping errors would result in incorrect haplotype structures.

\section{CONCLUSION}

This research represents the first time an accurate method has been developed to that can impute multi-allelic genotypes from bi-allelic data. Our results will have an immediate impact for livestock associations wishing to transition from MS- to SNPbased parentage verification. Additionally, the same methods can be used to impute MS alleles for studies that desire to combine data sets that include both MS and SNP genotypes. MShaplotype combinations that hold true across multiple phylogenetically unique cattle breeds (Decker et al., 2009; Table S2 in Supplementary Material) may represent historic MS-haplotypes. In theory, these MS-haplotypes could be used to accurately impute MS alleles in other Bos primigenius taurus breeds using the imputation (Table S2 in Supplementary Material) reported here.

For commercial application of this method, it is recommended that the SNP listed in Table S1 in Supplementary Material be used as a standard set thus allowing for easy imputation and standardization across labs and platforms. While these SNP are all present on the BovineHD assay, it is possible that some SNP will not be compatible with other commercial platforms. For haplotypes that are associated with $>1$ MS alleles, the percent of times each association is seen in the test population (Table S2 in Supplementary Material) could be incorporated in exclusion probabilities for parentage identification in multi-sire mating populations, in a manner similar to allele frequencies. A built in benefit of our method is that while the selected SNP provide excellent performance a reduced set of SNP could be used for imputation, although this could result a lower resolving power and accuracy. As more animals and breeds with accurate MS and SNP genotypes are collected a reanalysis with all of the data will result in increased resolving power, identification of rare MS-haplotype combinations, and potentially identification of combinations that can be used across breeds not tested in this study.

While MS are currently the international standard parentage verification for exported semen, this work represents a tool to quickly migrate toward SNP-based verification in one generation. Finally, these imputation methods can be implemented in any species with available high density SNP genotypes flanking MS allele genotypes. 


\section{ACKNOWLEDGMENTS}

Thank you to the USDA-ARS Animal Improvement Programs Laboratory for access to the BovineHD genotypes. To the USA Brown Swiss, Guernsey, Holstein, and Jersey Associations for MS genotypes. We also thank Jen McClure for her review of the manuscript. Research was funded by USDA-ARS CRIS 1265-31000098-00D. Mention of trade names or commercial products in this article is solely for the purpose of providing specific information

\section{REFERENCES}

Affymetrix Inc. (2011). Axiom GenomeWide BOS 1 Array Plate. Available at: http://media.affymetrix.com/ support/technical/datasheets/axiom gw_bos1_arrayplate_datasheet.pdf [accessed 5/16/2011].

Altschul, S. F., Gish, W., Miller, W., Myers, E. W., and Lipman, D. J. (1990). Basic local alignment search tool. J. Mol. Biol. 215, 403-410.

Anderson, E. C., and Garza, J. C. (2006). The power of single-nucleotide polymorphisms for large-scale parentage inference. Genetics 172, 2567-2582.

Barendse, W., Armitage, S. M., Kossarek, L. M., Shalom, A., Kirkpatrick, B. W., Ryan, A. M., Clayton, D., Li, L., Neibergs, H. L., Zhang, N., Grosse, W. M., Weiss, J., Creighton, P., McCarthy, F., Ron, M., Teale, A. J., Fries, R., McGraw, R. A., Moore, S. S., Georges, M., Soller, M., Womack, J. E., and Hetzel, D. J. S. (1994). A genetic linkage map of the bovine genome. Nat. Genet. 6, 227-235.

Baruch, E., and Weller, J. I. (2008). Estimation of the number of SNP genetic markers required for parentage verification. Anim. Genet. 39, 474-479.

Baumung, R., Simianer, H., and Hoffmann, I. (2004). Genetic diversity studies in farm animals - a survey. J. Anim. Breed. Genet. 121, 361-373.

Bishop, M. D., Kappes, S. M., Keele, J. W., Stone, R. T., Sunden, S. L., Hawkins, G. A., Toldo, S. S., Fries, R., Grosz, M. D., Yoo, J., and Beattie, C. W. (1994). A genetic linkage map for cattle. Genetics 136, 619-639.

Boichard, D., Chung, H., Dassonneville, R., David, X., Eggen, A., Fritz, S., Gietzen, K. J., Hayes, B. J., Lawley, C. T., Sonstegard, T. S., Van Tassell, C. P., Vanraden, P. M., ViaudMartinez, K. A., and Wiggans, G. R. (2012). Design of a bovine lowdensity SNP array optimized for imputation. PLoS ONE 7, e34130. doi:10.1371/journal.pone.0034130

Bonin, A., Bellemain, E., Bronken Eidesen, P., Pompanon, F.,
Brochmann, C., and Taberlet, P. (2004). How to track and assess genotyping errors in population genetics studies. Mol. Ecol. 13, 3261-3273.

Browning, S. R., and Browning, B. L. (2007). Rapid and accurate haplotype phasing and missing-data inference for whole-genome association studies by use of localized haplotype clustering. Am. J. Hum. Genet. 81, 1084-1097.

Davis, G. P., and Denise, S. K. (1998). The impact of genetic markers on selection. J. Anim. Sci. 76, 2331-2339. Mckay, S. D., Heaton, M. P., Chen, K. F., Cooper, A., Vilkki, J., Seabury, C. M., Caetano, A. R., Johnson, G. S., Brenneman, R. A., Hanotte, O., Eggert, L. S., Wiener, P., Kim, J. J., Kim, K. S., Sonstegard, T. S., Van Tassell, C. P., Neibergs, H. L., Mcewan, J. C., Brauning, R., Coutinho, L. L., Babar, M. E., Wilson, G. A., McClure, M. C., Rolf, M. M., Kim, J., Schnabel, R. D., and Taylor, J. F. (2009). Resolving the evolution of extant and extinct ruminants with high-throughput phylogenomics. Proc. Natl. Acad. Sci. U.S.A. 106, 18644-18649.

Ellegren, H. (1995). Mutation rates at porcine microsatellite loci. Mamm. Genome 6, 376-377.

Falconer, D. S., and Mackay, T. F. C. (1996). Introduction to Quantitative Genetics. Essex: Longman Group Ltd.

Gagneux, P., Boesch, C., and Woodruff, D. S. (1997). Microsatellite scoring errors associated with noninvasive genotyping based on nuclear DNA amplified from shed hair. Mol. Ecol. 6, 861-868.

Georges, M., and Massey, J. (1992). Polymorphic DNA Markers in Bovidae. Geneva: World Intellectual Property Organisation. WO Publ. No. 92/13102.

Gomez-Raya, L., Priest, K., Rauw, W. M., Okomo-Adhiambo, M., Thain, D., Bruce, B., Rink, A., Torell, R., Grellman, L., Narayanan, R., and Beattie, C. W. (2008). The value
Decker, J. E., Pires, J. C., Conant, G. C.,

and does not imply recommendation or endorsement by the US Department of Agriculture. The USDA is an equal opportunity provider and employer.

\section{SUPPLEMENTARY MATERIAL}

The Supplementary Material for this article can be found online at http://www.frontiersin.org/Livestock_Genomics/10.3389/fgene. 2012.00140/abstract

of DNA paternity identification in beef cattle: examples from Nevada's free-range ranches. J. Anim. Sci. 86, 17-24.

Harlizius, B., Lopes, M. S., Duijvesteijn, N., Van De Goor, L. H. P., Van Haeringen, W. A., Panneman, H., Guimaraes, S. E., Merks, J. W., and Knol, E. F. (2011). A single nucleotide polymorphism set for paternal identification to reduce teh costs of trait recording in commercial pig breeding. J. Anim. Sci. 1661-1668.

Heaton, M. P., Harhay, G. P., Bennett, G. L., Stone, R. T., Grosse, W. M., Casas, E., Keele, J. W., Smith, T. P. L., Chitko-Mckown, C. G., and Laegreid, W. W. (2002). Selection and use of SNP markers for animal identification and paternity analysis in U.S. beef cattle. Mamm. Genome 13, 272-281.

Illumina Inc. (2010). BovineHD Genotyping Beadchip. Available at: http://www.illumina.com/Docum ents/products/datasheets/datasheet bovineHD.pdf [accessed 6/12/2011 2011].

Illumina Inc. (2011). GoldenGate Bovine3K Genotyping BeadChip. Available at: http://www. illumina.com/Documents/products/ datasheets/datasheet_bovine3K.pdf [accessed 5/16/2012].

Israel, C., and Weller, J. I. (2000). Effect of misidentification on genetic gain and estimation of breeding value in dairy cattle populations. J. Dairy Sci. 83, 181-187.

Kelly, A. C., Mateus-Pinilla, N. E., Douglas, M., Shelton, P., and Novakofski, J. (2011). Microsatellites behaving badly: empirical evaluation of genotyping errors and subsequent impacts on population studies. Genet. Mol. Res. 10, 2534-2553.

Luikart, G., Zundel, S., Rioux, D., Miquel, C., Keating, K. A., Hogg, J. T., Steele, B., Foresman, K., and Taberlet, P. (2008). Low genotyping error rates and noninvasive sampling in bighorn sheep. J. Wildl. Manage. 72, 299-304.

Matukumalli, L. K., Lawley, C. T., Schnabel, R. D., Taylor, J. F., Allan, M. F.,
Heaton, M. P., O'connell, J., Moore, S. S., Smith, T. P., Sonstegard, T. S., and Van Tassell, C. P. (2009). Development and characterization of a high density SNP genotyping assay for cattle. PLoS ONE 4, e5350. doi:10.1371/journal.pone.0005350

McClure, M. C., Morsci, N. S., Schnabel, R. D., Kim, J. W., Yao, P., Rolf, M. M., Mckay, S. D., Gregg, S. J., Chapple, R. H., Northcutt, S. L., and Taylor, J. F. (2010). A genome scan for quantitative trait loci influencing carcass, post-natal growth and reproductive traits in commercial Angus cattle. Anim. Genet. 41, 597-607.

Senneke, S. L., Macneil, M. D., and Van Vleck, L. D. (2004). Effects of sire misidentification on estimates of genetic parameters for birth and weaning weights in Hereford cattle. J. Anim. Sci. 82, 2307-2312.

Steffen, P., Eggen, A., Dietz, A. B., Womack, J. E., Stranzinger, G., and Fries, R. (1993). Isolation and mapping of polymorphic microsatellites in cattle. Anim. Genet. 24, 121-124.

Sunden, S. L., Stone, R. T., Bishop, M. D., Kappes, S. M., Keele, J. W., and Beattie, C. W. (1993). A highly polymorphic bovine microsatellite locus: BM2113. Anim. Genet. 24, 69.

Tokarska, M., Marshall, T., Kowalczyk, R., Wojcik, J. M., Pertoldi, C., Kristensen, T. N., Loeschcke, V., Gregersen, V. R., and Bendixen, C. (2009). Effectiveness of microsatellite and SNP markers for parentage and identity analysis in species with low genetic diversity: the case of European bison. Heredity 103, 326-332.

Toldo, S. S., Fries, R., Steffen, P., Neibergs, H. L., Barendse, W., Womack, J. E., Hetzel, D. J., and Stranzinger, G. (1993). Physically mapped, cosmid-derived microsatellite markers as anchor loci on bovine chromosomes. Mamm. Genome 4, 720-727.

Vaiman, D., Mercier, D., MoazamiGoudarzi, K., Eggen, A., Ciampolini, R., Lepingle, A., Velmala R., Kaukinen, J., Varvio, 
S. L., Martin, P., Leveziel, H., and Guerin, G. (1994). A set of 99 cattle microsatellites: characterization, synteny mapping, and polymorphism. Mamm. Genome 5, 288-297.

Van Eenennaam, A. L., Weaber, R. L., Drake, D. J., Penedo, M. C., Quaas, R. L., Garrick, D. J., and Pollak, E. J. (2007). DNA-based paternity analysis and genetic evaluation in a large, commercial cattle ranch setting. J. Anim. Sci. 85, 3159-3169.
Zimin, A. V., Delcher, A. L., Florea, L., Kelley, D. R., Schatz, M. C., Puiu, D., Hanrahan, F., Pertea, G., Van Tassell, C. P., Sonstegard, T. S., Marcais, G., Roberts, M., Subramanian, P., Yorke, J. A., and Salzberg, S. L. (2009). A whole-genome assembly of the domestic cow, Bos taurus. Genome Biol. 10, R42.

Conflict of Interest Statement: The authors declare that the research was conducted in the absence of any commercial or financial relationships that could be construed as a potential conflict of interest.

Received: 29 May 2012; paper pending published: 03 July 2012; accepted: 12 July 2012; published online: 14 August 2012. Citation: McClure M, Sonstegard T, Wiggans $G$ and Van Tassell $C P$ (2012) Imputation of microsatellite alleles from dense SNP genotypes for parental verification. Front. Gene. 3:140. doi 10.3389/fgene.2012.00140
This article was submitted to Frontiers in Livestock Genomics, a specialty of Frontiers in Genetics.

Copyright (c) 2012 McClure, Sonstegard, Wiggans and Van Tassell. This is an open-access article distributed under the terms of the Creative Commons Attribution License, which permits use, distribution and reproduction in other forums, provided the original authors and source are credited and subject to any copyright notices concerning any third-party graphics etc. 